\title{
sciendo
}

\section{Muscle Thickness During Core Stability Exercises in Children and Adults}

\author{
by \\ Eleftherios Kellis ${ }^{1}$, Athanasios Ellinoudis ${ }^{1}$, Konstantina Intziegianni ${ }^{2}$, \\ Nikolaos Kofotolis ${ }^{1}$
}

\begin{abstract}
Core stability exercises are regular part of exercise programs for asymptomatic individuals across ages. The purpose of this study was to examine deep abdominal and multifidus muscle thickness in children and adults and to determine reliability of the rehabilitative ultrasound (RUSI) imaging. Transversus abdominis and lumbar multifidus thickness at rest and during core stability exercise were examined in pre-pubertal children $(N=23)$, adolescents $(N=$ 20), young adults $(N=21)$ and middle-aged adults $(N=22)$. Thirty-nine participants were re-tested one week after to establish reliability. Muscle thickness at rest was lower in children and adolescents compared with young and middleaged adults $(p<0.008)$. Young adults displayed the highest relative transversus abdominis thickness upon contraction $(p<0.008)$. Lumbar multfidus contraction thickness was greater in young-adults than middle-aged adults and prepubertal children $(p<0.008)$, but it was similar between young-adults and adolescents $(p>0.008)$. Reliability was high for both muscles $\left(\right.$ ICC $_{3,3}=0.76$ - 0.99). The age-related differences in muscle thickness indicate that core stability exercises may be beneficial for children and middle-aged adults.
\end{abstract}

Key words: childhood, musculoskeletal ultrasound, repeatability, core exercise, core stability.

\section{Introduction}

Core stability exercises have become popular for enhancing spinal stability and quality of life in various populations (Akuthota et al., 2008) as well as for sport performance and injury prevention (McGill, 2010). Common core stability exercises include the abdominal drawing-in maneuver (ADIM) and quadruped arm lifts (Akuthota et al., 2008). These exercises recruit deep muscles of the spine such as the lumbar multifidus (LM) and abdominal muscles such as the transversus abdominis (TRA) (Hodges, 1999).

Rehabilitation ultrasound imaging (RUSI) has been extensively used to image TRA and LM during various exercises (Teyhen et al., 2012, 2007). Specifically, this technique is used to examine muscle thickness and the cross-sectional area at rest and during contraction (Teyhen et al., 2012, 2007). Studies have shown that RUSI evaluation of TRA and LM morphology is valid compared with magnetic resonance imaging (Hides et al., 2006). Furthermore, by comparison to indwelling electromyography RUSI is considered a valid indicator of activation of specific muscles under specific conditions (Ferreira et al., 2011).

Although muscular performance improves as children grow, the factors which contribute to this increase are still under discussion (Herzog et al., 2011; Housh et al., 1994; Kellis et al., 2014; O'Brien et al., 2010a). Increases

\footnotetext{
1 - Laboratory of Neuromechanics, Department of Physical Education and Sport Science at Serres, Aristotle University of Thessaloniki, Serres, Greece.

2 - University Outpatient Clinic, Sports Medicine and Sports Orthopedics, University of Potsdam, Am Neuen Palais 10, Haus 12, 14469 Potsdam, Germany.
} 
in muscle strength have been associated with changes in muscle size (Housh et al., 1994), muscle moment-arms (O'Brien et al., 2010b), specific muscle tension ( $\mathrm{O}^{\prime}$ Brien et al., 2010c) and neuromuscular activation (Kellis et al., 2014; Maszczyk et al., 2016; Gołaś et al., 2017). With respect to muscle size, most studies examined large pennate muscles of the lower limbs (Herzog et al., 2011; Housh et al., 1994; Kellis et al., 2014; O'Brien et al., 2010a). Watanabe et al. (2013) reported that adult soccer players showed similar TRA thickness at rest compared with adolescent players. In contrast, adult players showed almost a 2.5 times greater contraction ratio during the draw-in-maneuver compared with adolescents. Based on these results, these authors commented that adolescents displayed "a developmental delay" of the TRA thickness and activation. However, three recent studies have shown a significant correlation between age and TRA thickness in adolescent athletes and non-athletes which is minimized when body mass effects are taken into consideration (Linek, 2018, 2017; Linek et al., 2017a). It appears that although age-related increases in TRA in adolescents are due to development of body mass, the capacity to contract TRA during exercise may be influenced by age.

The effects of aging on TRA and LM thickness have also been examined (Ikezoe et al., 2012; Mannion et al., 2008; Rankin et al., 2006; Sions et al., 2014; Stokes et al., 2005). In particular, some studies identified only a small correlation between age and TRA thickness in individuals aged from 22 to 62 years (Mannion et al., 2008; Rankin et al., 2006). Others found no difference in TRA (Ikezoe et al., 2012) and LM (Ikezoe et al., 2012; Stokes et al., 2005) thickness in young (aged 20.0 years) individuals compared to elderly participants (aged up to 85 years). Another study, however, reported greater LM thickness in older adults (60-85 years) than younger (18-40 years) ones (Sions et al., 2014). This was attributed to a greater body mass index in the elderly compared with adults (Sions et al., 2014). Nevertheless, young adults displayed greater LM thickness during exercise than older individuals (Sions et al., 2014), which probably indicates a better ability to increase contraction thickness compared to older individuals.

The reliability of measurements made with RUSI has been extensively investigated in adults (Hebert et al., 2009; Sions et al., 2015). A few research studies in young adolescents (Linek et al., 2015, 2014) reported that TRA thickness at rest displayed ICCs ranging from 0.68 of 0.97 , whilst the contraction thickness ratio (CTR), which is defined as muscle thickness during contraction relative to thickness at rest, displayed an ICC ranging from 0.81 to 0.85 . Others have reported greater reliability in older adolescents (Kim et al., 2018; Yang et al., 2014). Fewer studies reported on LM thickness in children and adolescents (Linek et al., 2018, 2014; Zapata et al., 2015). Particularly, Zapata et al. (2015) reported that LM thickness, determined at rest, showed good intra-rater (ICC3,3 $=0.83-0.99)$ and interrater reliability (ICC2,3 = 0.93-0.99) in adolescents with idiopathic scoliosis. It has been suggested that reliability of the RUSI measurements may vary with chronological age in adolescents due to the effects of psychological stress on US measurements (Linek et al., 2014, 2018). In addition, aging may have an effect on muscle and fascia echogenicity in the RUSI image thus increasing variation in thickness measurements (Sions et al., 2014).

Lack of sufficient coordination in core musculature can lead to decreased efficiency of movement and compensatory patterns, causing strain and overuse injuries (Akuthota et al., 2008). Early studies reported that thickness of deep abdominal and spinal muscles at rest and contraction are associated with low back pain and altered trunk motor control (Hodges and Richardson, 1999), although recent studies have shown opposite results (Gildea et al., 2013; Noormohammadpour et al., 2016). There is evidence that core stability exercises are beneficial for enhancing core stability and endurance in a school environment (Oliver et al., 2010). Examination of muscle thickness in children in comparison to older individuals may be particularly useful in defining the development of deep trunk musculature (Oliver et al., 2010; Zapata et al., 2015). There are suggestions that the examination may be more stressful for younger adolescents than older ones (Linek et al., 2018, 2014). Similarly, older individuals may have a lower thickness of LM when performing an exercise than young adults (Sions et al., 2014). To our knowledge, no other studies have examined 
differences in TRA and LM size at rest and contraction between pre-pubertal children, adolescents and adults. If the ability to recruit the core muscles varies across the life span, then structure and application of core stability programs may be age-dependent. Consequently, evaluation of deep abdominal and paraspinal muscle thickness using RUSI to monitor exercise efficiency is worthwhile. The aim of this study was twofold: first, to examine differences in of TRA and LM thickness measured using RUSI between pre-pubertal children, adolescents, young and middle-aged adults and, second, to examine the reliability of deep abdominal and multifidus muscle thickness in four different age groups. It was hypothesized that RUSI measurements would differ between the four different age groups and that RUSI measurements would be equally reliable for use in all groups.

\section{Methods}

\section{Participants}

Pre-pubertal children and adolescents were randomly selected from a local sports club, consisting of 46 pre-pubertal children and 39 adolescents. Young and middle-aged individuals were randomly selected from a local fitness club, from a total number of 68 young-adults (aged 18 to 25 years) and 48 middle-aged adults (aged between 45 and 55 years). To be included in this study, all participants followed regular fitness exercise no more than twice a week for the past two years and they had no previous experience with core-stability exercises. Furthermore, all participants had no history of spinal or pelvicrelated pain or related condition confirmed by medical examination. Initially all individuals were asked to take part in this study. A total of 86 individuals agreed to participate and they were divided into four age groups. Group 1 consisted of 23 pre-pubertal children (age $7.69 \pm 1.23$ years; body mass $39.4 \pm 2.9 \mathrm{~kg}$; body height $1.45 \pm 0.07$ m), Group 2 consisted of 20 adolescents (age 13.1 \pm 1.2 years; body mass $52.2 \pm 6.2 \mathrm{~kg}$; body height $1.54 \pm 0.06 \mathrm{~m})$, Group 3 consisted of 21 young adults (age $22.3 \pm 3.3$ years; body mass $66.8 \pm 5.8$ $\mathrm{kg}$; body height $1.72 \pm 0.08 \mathrm{~m}$ ) and Group 4 consisted of 22 middle-aged adults (age $54.0 \pm 4.1$ years; body mass $75.7 \pm 8.8 \mathrm{~kg}$; body height $1.74 \pm$ $0.07 \mathrm{~m}$ ). All participants and the parents or legal guardians of the children received verbal and written information about all procedures and gave their signed informed consent to participate. The study was approved by the local Medical Ethics Committee.

\section{Design}

All participants performed one measurement session of the TRA and LM thickness using RUSI. Of these, a total of 39 participants $(8$ pre-pubertal children, 9 adolescents, 14 young adults and 8 middle-aged adults) were re-tested in a follow-up session 7 days later.

\section{Measurements}

RUSI measurements were taken with the participants at rest and during contraction. Muscle thickness measurements were acquired using an ultrasonic apparatus (SSD-3500, ALOKA, Japan, frequency range 7-13 MHZ) with an electronic linear array probe of $10 \mathrm{MHz}$ wave frequency and a length of $6 \mathrm{~cm}$. The image signal was stored in a digital form through an analogue to digital converter (Canopus, Model ADVC 100, Grass Valley Inc., USA) at a rate of $25 \mathrm{~Hz}$. The primary investigator operated the ultrasound unit and did all the scanning for this study. Training of the primary investigator in the use of RUSI included a 2-month 1-1 practice with an experienced researcher (with 12-year experience) and a further 1-year practice with the specific measurements prior to the commencement of the study. Data were stored by the rest investigators. Therefore, the examiner was blinded to his own previous measurements.

\section{TRA measurements}

For assessment of TRA thickness, the protocol proposed by Hides et al. (2007) was used. Specifically, ultrasound measurements were performed, once at rest and second when the participant performed the ADIM. The participant was in a supine hook-lying position, with the knees in $60^{\circ}$ flexion and the hips in $30^{\circ}$ flexion (Figure 1) defined using an analogue goniometer. In this position, the first ultrasound measurement was taken. They were then asked to relax by breathing in and out, hold their breath out and then draw in their lower abdomen, for approximately $5 \mathrm{~s}$ without moving their spine. To ensure no spinal movement during the measurements a pressure biofeedback unit (Stabilizer pressure bio-feedback, Chattanooga, USA) was placed under the participant's lumbar 
spine and inflated to $40 \mathrm{mmHg}$. Any increase of pressure during this maneuver was considered as indicative of spinal movement and the participants were asked to repeat the trial. In this contraction state, the second US measurement was taken. All participants were provided a demonstration of each exercise. They then performed 5 familiarization trials under the supervision of the experimenter. Then, US evaluation took place. The main protocol included 3 drawing-in maneuvers, with a 2-min rest period between trials.

The US transducer was positioned on the anterolateral aspect of the abdominal wall, approximately $2.5 \mathrm{~cm}$ superior to the iliac crest and perpendicular to the mid-axillary line (Teyhen et al., 2012). To standardize the position of the transducer, the anterior fascial insertion of the TRA muscle was positioned approximately 2 $\mathrm{cm}$ from the medial edge of the US image when the participant was relaxed (Hides et al., 2007). As already stated, US images were obtained at rest and while holding the drawing-in maneuver (contraction) from the left side.

TRA muscle thickness was measured as the distance between the superior and inferior hyperechoic muscle fascias, at the middle of the US image. Measurements were conducted perpendicularly to the muscle fascias.

\section{LM evaluation}

For assessment of LM thickness, the prone upper extremity lifting task was used (Figure 1). Specifically, the participants assumed the prone position, with the right upper extremity abducted to approximately $120^{\circ}$, the elbow flexed to $90^{\circ}$, and pillow(s) placed under the abdomen to flatten the lumbar spine so that the lumbosacral junction angle was less than or equal to $10^{\circ}$ (confirmed by inclinometer measurement). The US probe was placed longitudinally along the midline of the spine, first over the L4 level, then moved laterally and tilted slightly medially until the L4-L5 facet joint was visualized. In this relaxed position, an US image was taken. The participant then performed the upper extremity lifting task by lifting the right arm off the table to induce a contraction of the left lumbar paraspinals. Low resistance was provided by using lifting weights equal to approximately 0.45 of body mass. The duration of the contraction was $8 \mathrm{~s}$ and the US image was also captured.
Following 5 familiarization trials, 3 contraction trials with a 2-min rest period in-between were performed. LM thickness measurement was expressed as the distance between the facet joint and the plane between the subcutaneous tissue and LM multifidus muscle (Figure 1).

All measurements were obtained via the electronic calipers of US software (displayed onscreen). The mean of 3 measures, which has been shown to reduce the standard error of the measurement by approximately $40 \%$ in adolescents (Linek et al., 2015) and 50\% in adults (Koppenhaver et al., 2009b), was used. All testing sessions were completed without any loss in participants or data errors.

An example of RUSI images at rest from each age group is presented in Figure 2. The dependent variables included TRA and LM thickness measured with the participants at rest and during contraction. In addition, the CTR was calculated as the percentage change in muscle thickness from rest to contraction.

\section{Statistical analysis}

Data were checked for normality using the Kolmogorov-Smirnov test. Analysis of variance (ANOVA) was used to compare body mass and height between groups. A one-way analysis of co-variance (ANCOVA) was used to examine group differences in each dependent variable, using body mass and height as covariates. If significant, a post-hoc analysis Tukey test was applied to determine significant differences between various pairs of means. The level of significance was set at $p<0.05$. Because of multiple comparisons performed on the same data, the level of significance was adjusted using the Bonferroni correction, with the accepted level of significance set at $p<0.008$.

An ICC was calculated to assess intraexaminer reliability $\left(\mathrm{ICC}_{3,3}\right)$ with a $95 \%$ confidence interval (CI: $95 \%$ ) based on the average of 3 measurements per session of each muscle. An ICC value $\leq 0.50$ was considered low, 0.50 to 0.75 moderate, $\geq 0.75$ good and $\geq 0.90$ excellent. Agreement between the measurements was examined using Bland-Altman analysis (Bias \pm limits of agreement (LoA) (Bland and Altman, 1986). Bias was calculated as the absolute difference in thickness $(\mathrm{mm})$ between test and retest sessions; values closer to 0 indicated greater agreement. The LoA was calculated as $1.96^{*} \mathrm{SD}$ 
representing a measure of random error between measurement sessions. In addition, the standard error of measurement (SEM) was calculated using the following formula:

\section{$\mathrm{SEM}=\mathrm{SD}^{*} \sqrt{1-I C C}$}

Test-Retest variability (TRV) was calculated as the absolute differences between the two measurements, divided by their average and expressed as a percentage (\%).

A Pearson's correlation test was used to examine the association between age and thickness at rest.

\section{Results}

Analysis of variance indicated that children had significantly less body mass and height than all other age groups $(p<0.05)$. Furthermore, adolescents showed lower body mass and height than young and middle-aged adults $(p<0.05)$. Finally, young-adults had lower body mass $(p<0.05)$, but similar height $(p>0.05)$ compared with middle-aged adults.

Age group differences

In all ANCOVA models, the F-ratios for the co-variates body mass and height were not statistically significant $(p>0.008)$. The one-way ANCOVA showed a statistically significant group difference in TRA values at rest $\left(\mathrm{F}_{3,82}=4.39, p<\right.$ $0.008)$, contraction $\left(\mathrm{F}_{3,82}=12.09, p<0.008\right)$ and CTR $\left(\mathrm{F}_{3,82}=7.10, p<0.008\right)$. Post-hoc Tukey tests indicated that young adults showed significantly greater TRA thickness compared with all groups $(p<0.008)$. Middle-aged adults showed significantly greater TRA thickness at rest and contraction compared with pre-pubertal children and adolescents $(p<0.008)$. The TRA CTR was similar in pre-pubertal children, adolescents and middle-aged adults $(p>0.008)$.

The ANCOVA showed statistically significant group differences in LM thickness at rest $\left(\mathrm{F}_{3,82}=9.47, p<0.008\right)$, contraction $\left(\mathrm{F}_{3,82}=8.19\right.$, $p<0.008)$ and CTR $\left(\mathrm{F}_{3,82}=3.58, p<0.008\right)$. Post-hoc Tukey tests indicated that young and middleaged adults showed significantly greater LM thickness at rest and contraction compared with pre-pubertal children and adolescents $(p<0.008)$. No other group differences in LM thickness at rest were observed ( $p>0.008)$. Finally, the LM CTR was significantly greater in young-adults compared with pre-pubertal children and middleaged adults $(p<0.008)$. No other group differences were found.

Reliability of TRA measures

The ICC values for TRA assessment ranged from 0.86 to 0.97 at rest, from 0.89 to 0.97 during ADIM and from 0.77 to 0.98 for the CTR. Adolescents presented the highest ICCs compared to the other groups (Table 2). In absolute terms, the SEM values ranged from 0.10 to $0.38 \mathrm{~mm}$ at rest, from 0.13 to $0.33 \mathrm{~mm}$ during ADIM and from 1.00 to $5.33 \%$ for CTR, with adolescents presenting the lowest SEM. The TRV values at rest ranged from 4.17 to $9.09 \%$, from 4.32 to $5.54 \%$ during ADIM and from 5.73 to $17.61 \%$ for the CTR, with adolescents demonstrating the lowest TRV values. Overall the systematic error was low in all groups ranging from 0.00 to $0.21 \mathrm{~mm}$ at rest, from 0.06 to $0.15 \mathrm{~mm}$ during ADIM and from 0.19 to $0.91 \%$ for the CTR. The random error range was 0.13-1.00 $\mathrm{mm}$ at rest, 0.18-0.72 during ADIM and $1.54-15.89 \%$ for the CTR, with young adults demonstrating the highest error.

Reliability of LM measures

The ICC values for LM assessment ranged from 0.77 to 0.99 at rest, from 0.81 to 0.99 during contraction and from 0.76 to 0.98 for CTR with adolescents presenting the highest ICCs (Table 3). In absolute terms, the SEM values range was 0.36 - $1.30 \mathrm{~mm}$ at rest, $0.38-1.92 \mathrm{~mm}$ during contraction and $1.14-3.78 \%$ for the CTR with adolescents presenting the lowest SEM. The TRV values range was $1.89-5.08 \%$ at rest, $1.67-4.51 \%$ during contraction and $7.08-13.11 \%$ for the CTR with adolescents demonstrating the lowest TRV. Overall, the systematic error was low in all groups ranging from $0.42-1.17 \mathrm{~mm}$ at rest, $0.11-0.91 \mathrm{~mm}$ during contraction and $0.03-1.29 \%$ for the CTR. The random error ranged between $0.42-3.20 \mathrm{~mm}$ at rest, 0.57-4.82 during contraction and for the CTR the range was $2.10-7.71 \%$ with pre-pubertal children and young adults demonstrating the highest random error value.

Correlation between age and thickness at rest

The Pearson's moment correlation coefficient between age and thickness at rest was 0.258 ( $p<$ $0.05)$ and $0.553(p<0.05)$ for TRA and LM thickness, respectively. 


\begin{tabular}{|c|c|c|c|c|}
\hline \multicolumn{3}{|c|}{$\begin{array}{c}\text { Table } \mathbf{1} \\
\text { Mean }( \pm S D) \text { of RUSI thickness values at rest and abdominal drawing- } \\
\text { in maneuver }(\text { ADIM) for transversus abdominis and contraction (for multifidus) } \\
\text { and contraction thickness ratio (CTR) for each age group. }\end{array}$} & \multicolumn{2}{|c|}{$\begin{array}{l}\text { Table } \mathbf{1} \\
\qquad \text { abdominal drawing- } \\
\text { ntraction (for multifidus) } \\
\text { ach age group. }\end{array}$} \\
\hline Variable & $\begin{array}{l}\text { Pre-pubertal } \\
\text { children }\end{array}$ & Adolescents & Young Adults & $\begin{array}{l}\text { Middle-aged } \\
\text { adults }\end{array}$ \\
\hline Sample (N) & 23 & 20 & 21 & 22 \\
\hline \multicolumn{5}{|c|}{ Transversus Abdominis } \\
\hline Rest (mm) & $2.94 \pm 0.56^{* \wedge}$ & $3.16 \pm 0.40^{* \wedge}$ & $4.12 \pm 0.78$ & $3.59 \pm 0.50^{*}$ \\
\hline $\operatorname{ADIM}(\mathrm{mm})$ & $3.56 \pm 0.71^{* \wedge}$ & $3.93 \pm 0.47^{* \wedge}$ & $5.54 \pm 0.94$ & $4.42 \pm 0.48^{*}$ \\
\hline CTR $(\%)$ & $21.17 \pm 5.27^{*}$ & $24.57 \pm 6.97^{*}$ & $37.69 \pm 11.22$ & $24.18 \pm 9.18^{*}$ \\
\hline \multicolumn{5}{|c|}{ Multifidus } \\
\hline Rest (mm) & $23.50 \pm 3.11^{* \wedge}$ & $23.74 \pm 3.41^{* \wedge}$ & $31.47 \pm 6.86$ & $32.83 \pm 5.06$ \\
\hline Contraction (mm) & $28.79 \pm 3.86^{* \wedge}$ & $29.41 \pm 3.57^{* \wedge}$ & $41.00 \pm 10.21$ & $39.77 \pm 5.47$ \\
\hline CTR (\%) & $22.58 \pm 3.36^{*}$ & $24.46 \pm 8.14$ & $30.01 \quad \pm 10.42$ & $21.70 \pm 7.19^{*}$ \\
\hline \multicolumn{5}{|c|}{$\begin{array}{l}\text { *Significantly different compared with young adults }(p<0.008) \\
\wedge \text { Significantly different compared with middle-aged adults }(p<0.008)\end{array}$} \\
\hline
\end{tabular}

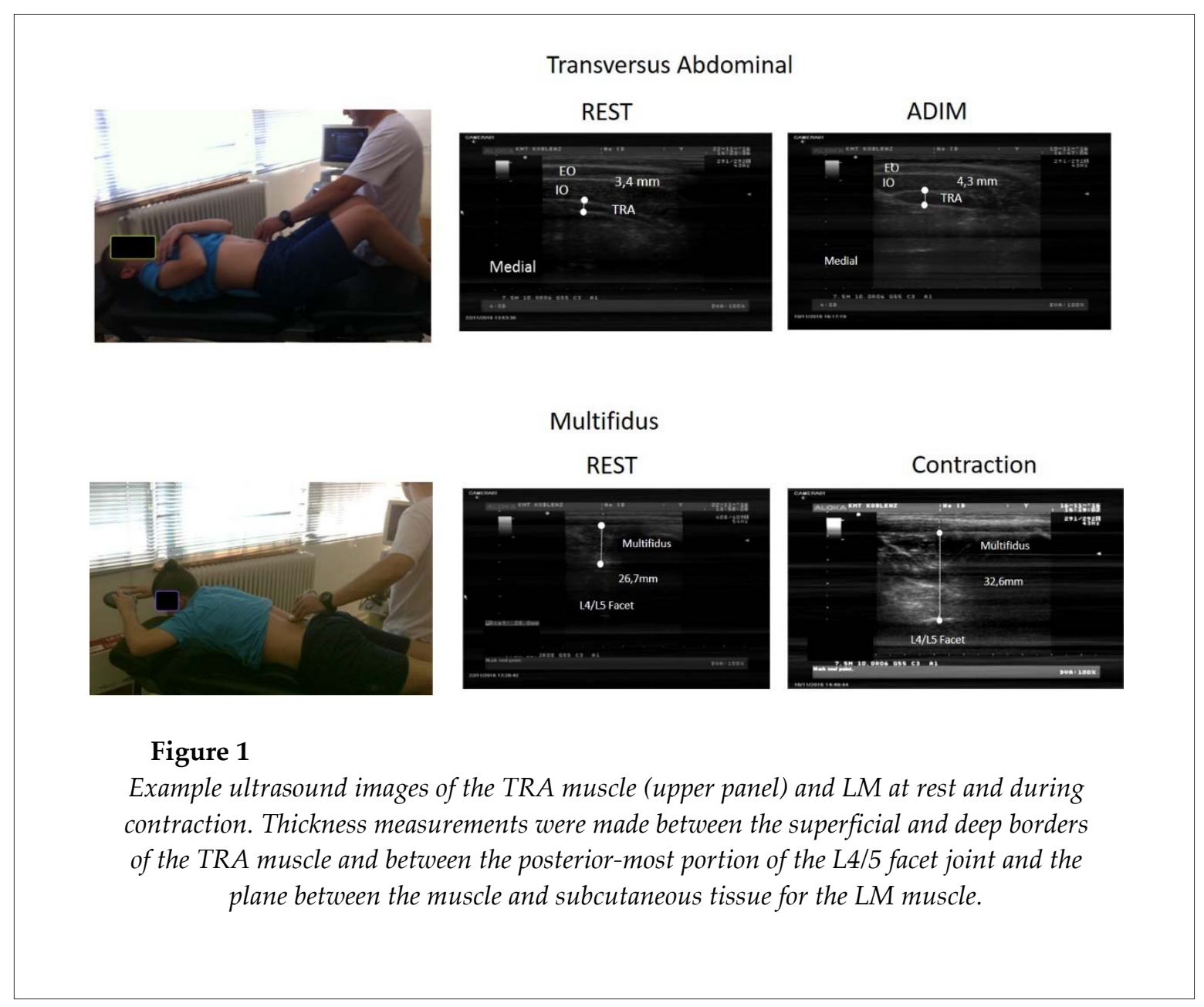




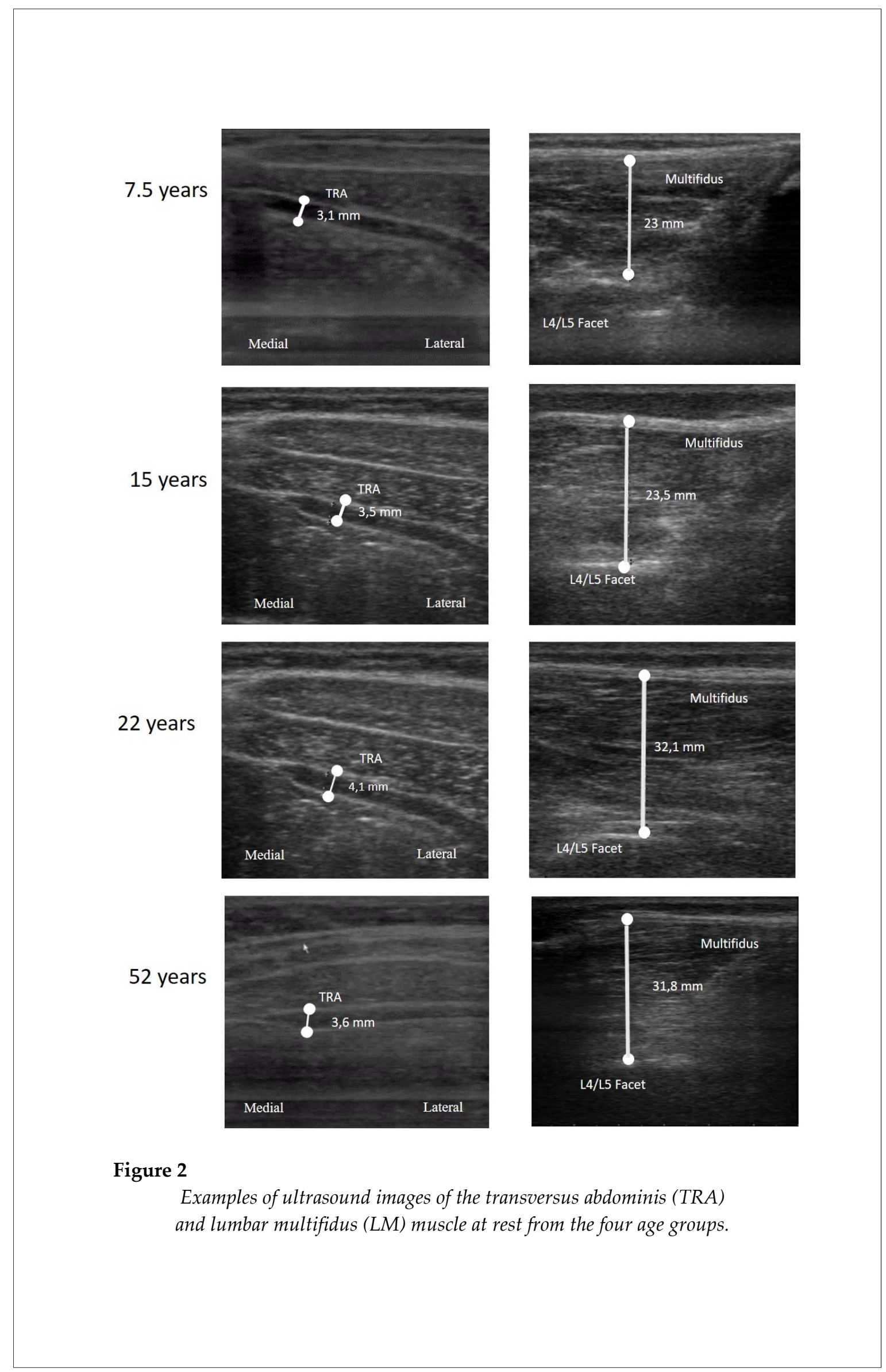


Table 2

Reliability values for transversus abdominis RUSI thickness values for all age groups.

\begin{tabular}{|c|c|c|c|c|c|c|}
\hline Muscle state & Test & Rest & $\mathrm{ICC}_{3,3}$ & SEM & Bias \pm LoA & TRV (\%) \\
\hline \multicolumn{7}{|c|}{ Pre-pubertal children $(\mathrm{N}=8)$} \\
\hline Rest (mm) & $3.02 \pm 0.36$ & $3.06 \pm 0.51$ & 0.86 & 0.14 & $0.14 \pm 0.47$ & $7.12 \pm 4.41$ \\
\hline Contraction (mm) & $3.81 \pm 0.40$ & $3.98 \pm 0.42$ & 0.89 & 0.13 & $0.15 \pm 0.43$ & $5.54 \pm 3.72$ \\
\hline CTR (\%) & $23.73 \pm 5.21$ & $22.82 \pm 3.05$ & 0.77 & 2.01 & $-0.91 \pm 5.83$ & $10.51 \pm 6.17$ \\
\hline \multicolumn{7}{|l|}{ Adolescents $(\mathrm{N}=9)$} \\
\hline Rest (mm) & $3.22 \pm 0.54$ & $3.28 \pm 0.57$ & 0.97 & 0.10 & $0.07 \pm 0.13$ & $4.17 \pm 1.6$ \\
\hline Contraction (mm) & $3.97 \pm 0.58$ & $4.06 \pm 0.64$ & 0.95 & 0.13 & $0.09 \pm 0.18$ & $4.47 \pm 2.10$ \\
\hline CTR (\%) & $23.86 \pm 5.97$ & $24.06 \pm 7.01$ & 0.98 & 1.00 & $0.19 \pm 1.54$ & $5.73 \pm 3.78$ \\
\hline \multicolumn{7}{|l|}{ Young adults $(\mathrm{N}=14)$} \\
\hline Rest (mm) & $3.92 \pm 1.07$ & $4.13 \pm 1.03$ & 0.87 & 0.38 & $0.21 \pm 1.00$ & $9.09 \pm 9.42$ \\
\hline Contraction (mm) & $5.40 \pm 1.36$ & $5.44 \pm 1.35$ & 0.94 & 0.33 & $0.01 \pm 0.72$ & $5.32 \pm 4.83$ \\
\hline CTR (\%) & $38.25 \pm 12.75$ & $37.71 \pm 10.83$ & 0.79 & 5.33 & $0.54 \pm 15.89$ & $17.61 \pm 13.48$ \\
\hline \multicolumn{7}{|c|}{ Middle aged adults $(\mathrm{N}=8)$} \\
\hline Rest (mm) & $3.62 \pm 0.47$ & $3.63 \pm 0.42$ & 0.92 & 0.12 & $0.00 \pm 0.38$ & $4.91 \pm 1.48$ \\
\hline Contraction (mm) & $4.37 \pm 0.50$ & $4.43 \pm 0.45$ & 0.91 & 0.14 & $0.06 \pm 0.40$ & $4.32 \pm 2.11$ \\
\hline CTR $(\%)$ & $20.97 \pm 5.14$ & $22.63 \pm 3.37$ & 0.81 & 1.39 & $0.81 \pm 3.76$ & $7.60 \pm 4.99$ \\
\hline
\end{tabular}

Measures of reliability: ICC: Intraclass Correlation Coefficient,

$S E M=$ standard error of measurement, Bias $\pm L o A=95 \%$ Limits of agreement,

$T R V=$ Test-Retest Variability. $C T R=$ contraction thickness ratio.

Table 3

Reliability values for multifidus RUSI thickness values for all age groups.

\begin{tabular}{|c|c|c|c|c|c|c|}
\hline Muscle state & Test & Rest & $\mathrm{ICC}_{3,3}$ & SEM & Bias \pm LoA & TRV $(\%)$ \\
\hline \multicolumn{7}{|c|}{ Pre-pubertal children $(\mathrm{N}=8)$} \\
\hline Contraction (mm) & $29.55 \pm 2.88$ & $30.00 \pm 3.48$ & 0.93 & 0.89 & $0.46 \pm 2.40$ & $3.34 \pm 1.82$ \\
\hline CTR (\%) & $22.59 \pm 2.81$ & $23.02 \pm 2.33$ & 0.79 & 1.15 & $-0.54 \pm 6.64$ & $11.61 \pm 7.99$ \\
\hline Rest (mm) & $23.85 \pm 4.33$ & $24.20 \pm 4.40$ & 0.99 & 0.36 & $0.34 \pm 0.42$ & $1.89 \pm 1.16$ \\
\hline Contraction (mm) & $29.27 \pm 4.11$ & $29.38 \pm 4.10$ & 0.99 & 0.38 & $0.11 \pm 0.57$ & $1.67 \pm 0.87$ \\
\hline CTR (\%) & $23.69 \pm 8.96$ & $22.40 \pm 8.49$ & 0.98 & 1.14 & $-1.29 \pm 2.10$ & $7.08 \pm 5.47$ \\
\hline \multicolumn{7}{|c|}{ Young adults $(\mathrm{N}=14)$} \\
\hline \multicolumn{7}{|c|}{ Middle aged adults $(\mathrm{N}=8)$} \\
\hline Rest (mm) & $33.91 \pm 2.21$ & $35.12 \pm 2.21$ & 0.77 & 1.03 & $1.17 \pm 2.12$ & $3.64 \pm 2.23$ \\
\hline Contraction (mm) & $40.40 \pm 3.52$ & $41.12 \pm 2.52$ & 0.91 & 0.91 & $0.72 \pm 2.41$ & $2.64 \pm 1.75$ \\
\hline CTR $(\%)$ & $19.59 \pm 4.45$ & $19.44 \pm 3.62$ & 0.76 & 1.94 & $-0.03 \pm 5.65$ & $13.11 \pm 10.59$ \\
\hline
\end{tabular}

Measures of reliability: ICC: Intraclass Correlation Coefficient,

$S E M=$ standard error of measurement, Bias $\pm L o A=95 \%$ Limits of agreement,

$T R V=$ Test-Retest Variability, $C T R=$ contraction thickness ratio 


\section{Discussion}

The main findings of this study were that: a) amongst the four groups, young adults showed the highest TRA and LM thickness at rest and contraction and the pre-pubertal children the lowest, b) the LM CTR was greater in youngadults and adolescents and lower in pre-pubertal children and adults, while the TRA CTR was greater in young-adults compared with all other age-groups, and c) RUSI assessment of TRA and $\mathrm{LM}$ at rest and during contraction displayed high reliability in all groups. This is the first study which examined RUSI assessment of both TRA and $\mathrm{LM}$ at rest and during contraction in various age categories.

Pre-pubertal children and adolescents showed lower TRA muscle thickness at rest and contraction than older age groups (Table 1). This is in line with previous suggestions that increases in lower limb muscle size can account for approximately $75 \%$ of differences in strength between children and adult males (Herzog et al., 2011; Housh et al., 1994; Kellis et al., 2014; O’Brien et al., 2010c). To our knowledge, no study has compared age differences in TRA thickness at rest and contraction including both children and middle-aged individuals. Two recent experiments in adolescents (Linek, 2017, 2018) have shown that oblique abdominal and TRA thickness adjusted for body mass has no association with age. Linek (2017) reported a significant correlation between TRA thickness and participants' age only in physically inactive adolescents. Our findings also showed a low association between age and TRA thickness across the whole sample, while there was no systematic difference in thickness between children and adolescents (Table 1). Watanabe et al. (2013) found no differences in TRA thickness at rest between adolescents and adults. Nevertheless, in the same study (Watanabe et al., 2013), adolescents displayed lower TRA thickness during contraction compared to adults, which is in line with the present findings.

Studies also reported no association between age and TRA thickness in healthy individuals aged from 20 to 80 years (Ikezoe et al., 2012; Mannion et al., 2008; Rankin et al., 2006). In our study, although TRA thickness and age had a low association, comparison between age groups showed that young adults displayed a greater CTR in comparison not only with children and adolescents, but also with middle-aged adults (Table 1). Since all participants had no specific experience with core stability exercises, we can suggest that growth and puberty as well as the aging process might have an effect on the ability to activate the deep spinal and abdominal muscles during contraction. Watanabe et al. (2013) attributed the difference in the CTR between adolescents and adults to an immature contraction of the TRA and the trunk musculature, in general. This conclusion was based on previous observations that development of the spinal column was completed towards the end of the developmental period (Tanner et al., 1982). In fact, our results indicate that prepubertal children showed marginally smaller TRA thickness than adolescents confirming the above suggestion.

Group differences in LM thickness were generally similar to those reported for TRA measurements (Table 1). To our knowledge, there are no data regarding age-related differences in LM thickness at rest and during contraction during core stability exercises using ultrasound including young children and middle-aged adults. There was a moderate correlation between LM thickness at rest and age for the whole sample. This is not in line with previous studies which focused only on older adults and showed no association between age and LM thickness (Ikezoe et al., 2012; Stokes et al., 2005). One may suggest that after reaching adulthood, the development of thickness with age may not be linear, at least within the range of age categories examined in the present study. However, more research including a sample from a wider age range is necessary before drawing generalized conclusions. Comparison of LM thickness between young ( $>20$ years) and older ( $>60$ years) individuals has shown conflicting results as some investigators reported no differences (Ikezoe et al., 2012), but others reported greater thickness in older individuals than younger ones (Sions et al., 2014). However, two findings related to LM thickness are noteworthy. First, although LM thickness at rest was lower in adolescents than young-adults, the LM thickness contraction ratio (CTR) was similar between these two groups. This indicates that adolescents showed a similar (relative) ability to recruit the LM muscle to young adults. Second, although LM thickness at 
rest was similar in the middle-aged and youngadult groups, the LM CTR was lower in middleaged adults compared with young adults (Table 1). A similar observation has been reported by Sions et al. (2014). Consequently, it appears that the high LM thickness at rest displayed by the middle-aged group was not translated into a high CTR of the same muscle during an exercise involving submaximal LM contraction. This may be due to aging effects on muscle mass or the presence of intramuscular fat secondary to aging (Hadar et al., 1983; Sions et al., 2015). Collectively, within the limitations of the cross-sectional design of the present study, the above findings indicate that age-group differences in LM thickness at rest were not accompanied by similar differences in LM contractile thickness during exercise.

Middle-aged adults showed greater TRA and LM thickness at rest and during contraction compared with children and adolescents (Table 1). One may suggest that this is due to immature development of muscles in young children. This is based on three factors: first, that body mass was taken into consideration for group comparisons, second, there were no differences in thickness between the two adult groups (middle-aged and young adults) and, finally, that middle-aged adults may display greater US thickness due to greater fat or connective tissue (Sions et al., 2015; Stokes et al., 2005). It is interesting, however, that neither the TRA nor the LM CTR differed between these three age groups (Table 1). This indicates that middle-aged individuals could not achieve a greater CTR of TRA and LM during submaximal exercises, despite having greater absolute thickness than children and adolescents. Within the limitations of ultrasound methodology applied in the present study, this implies that the ability to recruit core muscles increases from childhood to early adulthood, but it decreases in middle-aged individuals. One might also consider that these exercises are voluntary activities and therefore, muscle recruitment may be influenced by various factors, such as the ability of the participant to understand the instruction, fear of pain and variability in performance (Teyhen et al., 2012; Van et al., 2006). These factors may have greater influence on the performance of core exercises in children and middle-aged adults than young adults.

Intra-examiner comparisons of TRA thickness showed high reliability, especially for adolescents (Table 2). These findings are in agreement with previous studies which investigated asymptomatic adults (Hebert et al., 2009; Koppenhaver et al., 2009a; Larivière et al., 2013; McPherson and Watson, 2012) and support the hypothesis that RUSI measurements are adequately reliable for research and clinical use in children and adolescents. Linek et al. (2015) reported similar ICC (0.95) for RUSI measurement of TRA thickness during the straight leg raising test at rest whilst the ICC for the CTR was somewhat lower (0.81 to 0.85$)$ than the ICC values reported in the present study. The same research group (Linek et al., 2014) found ICCs ranging from 0.68 to 0.97 when TRA thickness was assessed from a supine position at rest in 10-11 year-olds, while ICCs for 12-15 year-old adolescents were somewhat greater. Therefore, it appears that the results of the present study extend these findings to TRA measurements when children of different age perform the ADIM.

The results of the present study showed a high ICC (>0.95) of LM thickness in adolescents and moderate reliability (ICC > 0.79) in the remaining groups. This is in agreement with Zapata et al. (2015) who reported that LM thickness in children measured at rest showed good intra-rater (ICC $=0.83-0.99)$ and high interrater reliability $(\mathrm{ICC}=0.93-0.99)$. Similar results were reported for young and older adults (Sions et al., 2015). The SEM for rest values in adolescents was low $(<0.36 \mathrm{~cm})$ whilst for the other groups it was generally greater $(<1.29 \mathrm{~cm})$. This may be due to a greater effect on connective tissue and bone on ultrasound image echogenicity of the LM muscle which is often observed in older adults (Stokes et al., 2005). Nevertheless, it appears that performing this specific task is accompanied by test-retest variation in LM thickness at rest and during contraction of no more than $5 \%$ which is very similar to that reported by previous studies (Koppenhaver et al., 2009a, 2009b; Sions et al., 2014; Wong et al., 2013). Consequently, our results indicated that RUSI could be applied to quantify the LM muscle CTR during core stability exercises, especially in adolescents.

All indices of reliability were lower for the CTR than thickness evaluated at rest or during contraction (Tables 2-3). This led to greater 
variability of this variable across testing sessions which reached almost $17 \%$ for the TRA evaluation in young adults (Table 2). It appears that changes in the CTR after a core stability exercise program in children should exceed 7\% for TRA and $6 \%$ for $\mathrm{LM}$ of the baseline test value so that the examiner is $95 \%$ confident that a true change occurred. For young adults, the corresponding values are greater, reaching $17 \%$ of baseline value for TRA. Similar results have been reported for older individuals (Sions et al., 2015). Based on these findings a cautious interpretation of CTR changes following exercise intervention programs is recommended.

The applications of RUSI for the assessment of TRA and LM thickness shows some limitations. First, all measurements were performed by the same examiner which affects their generalizability to other examiners. Since the procedures followed in the present study were carefully applied based on previous protocols, it is very likely that trained examiners with similar experience would produce similar results. Second, RUSI examination of one side was examined. Third, only asymptomatic individuals were examined; and thus, its application in the clinical field should be further investigated. Finally, in this study, measures were made on the US machine during imaging acquisition, rather than using a separate imaging analysis software. This might limit the RUSI analysis precision, but the reliability measurements reflect real-life application of RUSI.

The results of this study were obtained from healthy individuals who were involved in sport and physical activity less than twice a week. It is possible that TRA and LM thickness profiles differ in less or more physically active individuals (Linek, 2017). Furthermore, in the present study muscle thickness was obtained only from one side. Hence, any asymmetries in muscle contraction between sides were not taken into consideration. Finally, muscle thickness at rest and during contraction may vary between genders (Linek et al., 2017b), but such differences were not examined in this study.

The results have some implications for monitoring core stability exercise program effectiveness. First, the observation that TRA and LM thickness varies amongst groups indicates that interpretation of RUSI results should be agespecific. In this way, any muscle atrophy can be identified and treated accordingly using individualized exercise interventions. Second, exercise dosage may differ between young children, adults and older individuals, as their ability to recruit the core stability musculature during the same exercise may not be the same. Furthermore, exercise programs which aim to improve the technique of performing core exercises may be particularly beneficial for young children or individuals who have no experience in performing such movements. This may be beneficial in reducing the risk for musculoskeletal injuries (Huxel Bliven and Anderson, 2013), although clearly more research in this area is necessary.

In conclusion, the reduced thickness displayed by children and adolescents in this study does not imply muscle atrophy as it may be part of the growth and development. Since the ability to activate the deep trunk musculature depends on proprioception, motor learning ability and the respiratory pattern (Teyhen et al., 2007), the lower CTR displayed by children and middleaged adults compared to young adults indicates that children may not fully activate their muscles during a typical core stability exercise. Consequently, core stability programs may be beneficial for improving core stability in children and middle-aged adults.

\section{References}

Akuthota V, Ferreiro A, Moore T, Fredericson M. Core stability exercise principles. Curr Sports Med Rep, 2008; 7: 39-44

Bland JM, Altman DG. Statistical methods for assessing agreement between two methods of clinical measurement. Lancet, 1986; i: 307-310

Ferreira PH, Ferreira ML, Nascimento DP, Pinto RZ, Franco MR, Hodges PW. Discriminative and reliability analyses of ultrasound measurement of abdominal muscles recruitment. Man Ther, 2011; 16: 463-469 
Gildea JE, Hides JA, Hodges PW. Morphology of the abdominal muscles in ballet dancers with and without low back pain: A magnetic resonance imaging study. J Sci Med Sport, 2013; 17: 452-6

Gołaś A, Maszczyk A, Pietraszewski P, Stastny P, Tufano JJ, Zając A. Effects of Pre-exhaustion on the Patterns of Muscular Activity in the Flat Bench Press. J Strength Cond Res, 2017; 31(7): 1919-1924

Hadar H, Gadoth N, Heifetz M. Fatty replacement of lower paraspinal muscles: Normal and neuromuscular disorders. Am J Neuroradiol, 1983; 141: 895-8

Hebert JJ, Koppenhaver SL, Parent EC, Fritz JM. A systematic review of the reliability of rehabilitative ultrasound imaging for the quantitative assessment of the abdominal and lumbar trunk muscles. Spine, 2009; 34: E848-856

Herzog W, Sartorio A, Lafortuna CL, Kanehisa H, Fukunaga T, Dotan R, Falk B, Wood LE, Tonson A, Le Fur Y, Cozzone PJ, Bendahan D, Tolfrey K, Morse CI, O’Brien TD, Reeves ND, Baltzopoulos V, Jones DA, Maganaris CN, Lambertz D, Grosset JF, Perot C. Commentaries on Viewpoint: can muscle size fully account for strength differences between children and adults? J Appl Physiol, 2011; 110: 1750-3

Hides J, Wilson S, Stanton W, McMahon S, Keto H, McMahon K, Bryant M, Richardson C. An MRI investigation into the function of the transversus abdominis muscle during "drawing-in" of the abdominal wall. Spine, 2006; 31: E175-8

Hides J a, Miokovic T, Belavý DL, Stanton WR, Richardson C a. Ultrasound imaging assessment of abdominal muscle function during drawing-in of the abdominal wall: an intrarater reliability study. $J$ Orthop Sports Phys Ther, 2007; 37: 480-486

Hodges PW. Is there a role for transversus abdominis in lumbo-pelvic stability? Man Ther 1999; 4: 74-86

Hodges PW, Richardson CA. Altered trunk muscle recruitment in people with low back pain with upper limb movement at different speeds. Arch Phys Med Rehabil, 1999; 80: 1005-1012

Housh DJ, Donlin P, Housh TJ, Weir JP, Weir LL, Stout JR, Johnson GO. Isokinetic peak torque and crosssectional area of the quadriceps. Isokinet Exerc Sci, 1994; 4: 3-7

Huxel Bliven KC, Anderson BE. Core stability training for injury prevention. Sports Health, 2013; 5: 514-22

Ikezoe T, Mori N, Nakamura M, Ichihashi N. Effects of age and inactivity due to prolonged bed rest on atrophy of trunk muscles. Eur J Appl Physiol, 2012; 112: 43-48

Kellis E, Mademli L, Patikas D, Kofotolis N. Neuromuscular interactions around the knee in children, adults and elderly. World J Orthop, 2014; 5: 469-485

Kim DK, Kim CY, Lee BK, Seo D. A comparison of ultrasonography measurement on the abdominal muscle thickness between adolescent idiopathic scoliosis and healthy subjects. J Back Musculoskelet Rehab, 2018; 31: 65-74

Koppenhaver SL, Hebert JJ, Fritz JM, Parent EC, Teyhen DS, Magel JS. Reliability of rehabilitative ultrasound imaging of the transversus abdominis and lumbar multifidus muscles. Arch Phys Med Rehabil, 2009a; 90: 87-94

Koppenhaver SL, Parent EC, Teyhen DS, Hebert JJ, Fritz JM. The effect of averaging multiple trials on measurement error during ultrasound imaging of transversus abdominis and lumbar multifidus muscles in individuals with low back pain. J Orthop Sports Phys Ther, 2009b; 39: 604-611

Larivière C, Gagnon D, De Oliveira E, Henry SM, Mecheri H, Dumas JP. Reliability of Ultrasound Measures of the Transversus Abdominis: Effect of Task and Transducer Position. PM and R, 2013; 5: 104-113

Linek $P$. The importance of body mass normalisation for ultrasound measurements of the morphology of oblique abdominis muscles: the effect of age, gender, and sport practice. Folia Morphol, 2018; 77: 123130

Linek P. The importance of body mass normalisation for ultrasound measurement of the transversus abdominis muscle: The effect of age, gender and sport practice. Musculoskelet Sci Pract, 2017; 28: 65-70

Linek P, Klepek A, Wolny T, Mikołajowski G. Reliability of the lateral abdominal muscle thickness measurements in idiopathic scoliosis patients. Musculoskelet Sci Pract, 2018; 38: 151-154

Linek P, Saulicz E, Wolny T, Mysliwiec A. Intra-rater reliability of B-mode ultrasound imaging of the abdominal muscles in healthy adolescents during the active straight leg raise test. $P M$ and $R, 2015 ; 7$ : 53-59

Linek P, Saulicz E, Wolny T, Myśliwiec A. Body Mass Normalization for Ultrasound Measurements of Adolescent Lateral Abdominal Muscle Thickness. J Ultrasound Med, 2017a; 36: 775-782 
Linek P, Saulicz E, Wolny T, Myśliwiec A. Assessment of the abdominal muscles at rest and during abdominal drawing-in manoeuvre in adolescent physically active girls: A case-control study. J Sport Health Sci, 2017b; 6: 118-124

Linek P, Saulicz E, Wolny T, Myśliwiec A. Reliability of B-mode sonography of the abdominal muscles in healthy adolescents in different body positions. J Ultrasound Med, 2014; 33: 1049-56

Mannion AF, Pulkovski N, Toma V, Sprott H. Abdominal muscle size and symmetry at rest and during abdominal hollowing exercises in healthy control subjects. J Anat, 2008; 213: 173-182

Maszczyk A, Golas A, Czuba M, Krol H, Wilk M, Stastny P, Goodwin J, Kostrzewa M, Zajac A. EMG Analysis and Modelling of Flat Bench Press Using Artificial Neural Networks. SAJRSPER. 2016; 38(1): 91-103.

McGill S. Core Training: Evidence Translating to Better Performance and Injury Prevention. Strength Cond J, 2010; 32: 33-46

McPherson SL, Watson T. Reproducibility of Ultrasound Measurement of Transversus Abdominis During Loaded, Functional Tasks in Asymptomatic Young Adults. PM and R, 2012; 4: 402-412

Noormohammadpour P, Hosseini Khezri A, Linek P, Mansournia MA, Hassannejad A, Younesian A, Farahbakhsh F, Kordi R. Comparison of Lateral Abdominal Muscle Thickness and Cross Sectional Area of Multifidus in Adolescent Soccer Players with and without Low Back Pain: A Case Control Study. Asian J Sports Med, 2016; 7: e38318

O'Brien TD, Reeves ND, Baltzopoulos V, Jones DA, Maganaris CN. Muscle-tendon structure and dimensions in adults and children. J Anat, 2010a; 216: 631-642

O'Brien TD, Reeves ND, Baltzopoulos V, Jones DA, Maganaris CN. Mechanical properties of the patellar tendon in adults and children. J Biomech, 2010b; 43: 1190-1195

O'Brien TD, Reeves ND, Baltzopoulos V, Jones DA, Maganaris CN. In vivo measurements of muscle specific tension in adults and children. Exp Physiol, 2010c; 95: 202-210

Oliver G, Adams-Blair H, Dougherty C. Implementation of a Core Stability Program for Elementary School Children. Athl Train Sport Health Care, 2010; 2: 261-266

Rankin G, Stokes M, Newham DJ. Abdominal muscle size and symmetry in normal subjects. Muscle Nerve, 2006; 34: 320-6

Sions JM, Velasco TO, Teyhen DS, Hicks GE. Reliability of ultrasound imaging for the assessment of lumbar multifidi thickness in older adults with chronic low back pain. J Geriatr Phys Ther, 2015; 38: 33-9

Sions JM, Velasco TO, Teyhen DS, Hicks GE. Ultrasound imaging: intraexaminer and interexaminer reliability for multifidus muscle thickness assessment in adults aged 60 to 85 years versus younger adults. J Orthop Sports Phys Ther, 2014; 44: 425-34

Stokes M, Rankin G, Newham DJ. Ultrasound imaging of lumbar multifidus muscle: Normal reference ranges for measurements and practical guidance on the technique. Manual Therapy, 2005; 10: 116-126

Tanner JM, Hayashi T, Preece M a, Cameron N. Increase in length of leg relative to trunk in Japanese children and adults from 1957 to 1977: comparison with British and with Japanese Americans. Ann Hum Biol, 1982; 9: 411-23

Teyhen DS, Bluemle LN, Dolbeer JA, Baker SE, Molloy JM, Whittaker J, Childs JD. Changes in lateral abdominal muscle thickness during the abdominal drawing-in maneuver in those with lumbopelvic pain. J Orthop Sports Phys Ther, 2009; 39: 791-798

Teyhen DS, Childs JD, Stokes MJ, Wright AC, Dugan JL, George SZ. Abdominal and lumbar multifidus muscle size and symmetry at rest and during contracted States. Normative reference ranges. J Ultrasound Med, 2012; 31: 1099-1110

Teyhen DS, Gill NW, Whittaker JL, Henry SM, Hides J a, Hodges P. Rehabilitative ultrasound imaging of the abdominal muscles. J Orthop Sports Phys Ther, 2007; 37: 450-466

Van K, Hides JA, Richardson CA. The use of real-time ultrasound imaging for biofeedback of lumbar multifidus muscle contraction in healthy subjects. J Orthop Sports Phys Ther, 2006; 36: 920-925

Wallwork TL, Stanton WR, Freke M, Hides JA. The effect of chronic low back pain on size and contraction of the lumbar multifidus muscle. Man Ther, 2009; 14: 496-500

Watanabe H, Abe H, Tojima M, Yoshimoto M, Takahira N, Torii S. Ultrasonography of the deep abdominal muscles at rest and during the drawing-in maneuver: A comparative study of Japanese adolescent vs. 
adult soccer players. Isokinet Exerc Sci, 2013; 21: 187-193

Wong AYL, Parent EC, Kawchuk GN. Reliability of 2 ultrasonic imaging analysis methods in quantifying lumbar multifidus thickness. J Orthop Sports Phys Ther, 2013; 43: 251-62

Yang HS, Yoo JW, Lee BA, Choi CK, You JH. Inter-tester and intra-tester reliability of ultrasound imaging measurements of abdominal muscles in adolescents with and without idiopathic scoliosis: a casecontrolled study. Bio-Med Mater Eng, 2014; 24: 453-8

Zapata KA, Wang-Price SS, Sucato DJ, Dempsey-Robertson M. Ultrasonographic measurements of paraspinal muscle thickness in adolescent idiopathic scoliosis: a comparison and reliability study. Pediatr Phys Ther, 2015; 27: 119-25

\section{Corresponding author:}

Eleftherios Kellis, Ph.D.

Professor in Kinesiology

TEFAA Serres

Agios Ioannis

62100 Serres

Greece

E-mail: ekellis@phed-sr.auth.gr 